\title{
A TWO-DIMENSIONAL OPERATOR IDENTITY WITH APPLICATION TO THE CHANGE OF SIGN IN SUMS OF RANDOM VARIABLES $\left({ }^{1}\right)$
}

\author{
BY \\ GLEN BAXTER
}

1. Introduction. The topic of fluctuations of partial sums of independent random variables has received considerable attention in recent years (see $[1 ; 2 ; 3 ; 5 ; 6])$. In this paper we investigate still another fluctuation problem, namely, that of the number of changes of sign in the sequence of partial sums of random variables. Some results already obtained in this direction can be found in [1]. The precise statement of the problem on change of sign that we consider here is given at the beginning of $\$ 3$.

In $\S 2$, we develop the analytical tools which are needed for $\S 3$. We wish to point out one aspect of the analysis to which only minor attention has been paid. That is, up to a point the results are far more general than the arguments normally used to derive them. Let us consider a simple example. This will help to illustrate the point of view adopted in $\$ 2$. This example arises in the evaluation of the distribution of the number of positive partial sums $S_{0}=0, S_{1}, S_{2}, \cdots, S_{n}$ of a sequence $\left\{X_{k}\right\}$ of independent, identically distributed random variables. Considerable attention is given to this problem in the references listed above.

Let $A$ be the space of functions

$$
\phi=\sum_{k=-\infty}^{\infty} A_{k} e^{i k t} \text { with }\|\phi\|=\sum_{k=-\infty}^{\infty}\left|A_{k}\right|<\infty,
$$

and let

$$
\phi^{+}=\sum_{k=1}^{\infty} A_{k} e^{i k t}, \quad \phi^{-}=\sum_{k=-\infty}^{0} A_{k} e^{i k t} .
$$

Let $A^{+}$and $A^{-}$denote the space of functions $\phi^{+}$and $\phi^{-}$, respectively, as $\phi$ ranges over $A$. The elements of $A^{+}$are bounded and analytic in $t$ in the upper half-plane, and the elements of $A^{-}$are bounded and analytic in $t$ in the lower half-plane. For a fixed element $\psi$ in $A$, we now define the sequence $\left\{\phi_{k}\right\}$ by

Presented to the Society, January 22, 1959 under the title On the number of changes in sign; received by the editors October 26, 1959.

(1) This research was supported in part by the United States Air Force through the Air Force Office of Scientific Research of the Air Research and Development Command, under contract No. AF 18 (603)-30. Reproduction in whole or in part is permitted for any purpose of the United States Government. 


$$
\begin{aligned}
\phi_{0} & =1, \\
\phi_{n+1} & =u\left(\psi \phi_{n}\right)^{+}+\left(\psi \phi_{n}\right)^{-} .
\end{aligned}
$$

It can be shown that for $|s|<1 /\|\psi\|$

$$
\phi=\sum_{n=0}^{\infty} \phi_{n} s^{n}
$$

converges to an element of $A$ and is the unique solution of

$$
\phi=1+u s(\psi \phi)^{+}+s(\psi \phi)^{-} .
$$

Since $\phi=\phi^{+}+\phi^{-}$, Equation (1.2) can be rewritten

$$
[(1-u s \psi) \phi]^{+}=[1-(1-s \psi) \phi]^{-} .
$$

A typical argument goes as follows: the function on the left in (1.3) is analytic and bounded in the upper half-plane while the function on the right is analytic and bounded in the lower half-plane. Since the only bounded entire function is a constant, each side of (1.3) is a constant. By the definition of + , the constant must be zero. This means

$$
\begin{array}{r}
(1-u s \psi) \phi \in A^{-}, \\
(1-s \psi) \phi-1 \in A^{+} .
\end{array}
$$

If we put $P=(1-s \psi) \phi$ and $Q=(1-u s \psi) \phi$, then $P-1$ is in $A^{+}$and $Q-1$ is in $A^{-}$. It is possible to find an explicit solution to (1.2) on the basis of (1.4). In fact, (see $[2 ; 3]$ )

$$
\phi=\exp \{[-\log (1-s \psi)]-+[-\log (1-u s \psi)]+\} .
$$

At any rate, it is easy to show that (1.4) uniquely determines $\phi$.

Let us now note the triviality (for $|s|$ sufficiently small)

$$
\frac{P}{Q}=\frac{1-s \psi}{1-u s \psi},\left(P-1 \text { in } A^{+} \text {and } Q-1 \text { in } A^{-}\right) .
$$

Actually, (1.5) contains as much information as (1.4) in the sense that it uniquely determines $P$ and $Q$. However, relation (1.5) is extremely general and the method we have used above to derive (1.5) from (1.2) is completely beside the point. In fact, using (1.2)

and

$$
P=(1-s \psi) \phi=\phi-s \psi \phi=1+u s(\psi \phi)^{+}-s(\psi \phi)^{+},
$$

$$
Q=(1-u s \psi) \phi=\phi-u s \psi \phi=1-u s(\psi \phi)^{-}+s(\psi \phi)^{-} .
$$

Thus, $P-1$ is in $A^{+}$and $Q-1$ is in $A^{-}$, and (1.5) is true simply if $1 / Q$ is in $A$. Only the boundedness and linearity of + and the fact that $\phi^{-}=\phi-\phi^{+}$is necessary to derive (1.5). We interpret this observation to mean that unique- 
ness of the solution of (1.5) is the crucial question in this example. In general we would not expect an equation like (1.5) to uniquely determine $P$ and $Q$.

In the next section, we construct a two dimensional analogue of (1.5) in a quite generalized setting. We then give a condition to insure uniqueness of the factors.

2. A two-dimensional identity. Let $A$ denote a commutative Banach algebra of elements $\phi$ on which a bounded, linear operator + is defined taking $A$ into $A$. Define $\phi^{-}=\phi-\phi^{+}$and let $A^{+}$and $A^{-}$denote, respectively, the totality of elements $\phi^{+}$and $\phi^{-}$as $\phi$ ranges over $A$. Since + is a bounded operator, - will also be a bounded operator. Let $N$ be the maximum of the bounds of + and - .

For the arbitrary but fixed elements $\psi_{1}, \psi_{2}, \psi_{3}$, and $\psi_{4}$ in $A$, we define four sequences $\left\{\phi_{n}\right\},\left\{\chi_{n}\right\},\left\{\tilde{\phi}_{n}\right\}$, and $\left\{\tilde{\chi}_{n}\right\}$ by

$$
\begin{aligned}
\phi_{0} & =\tilde{\chi}_{0}=0, \quad \tilde{\phi}_{0}=\chi_{0}=1, \\
\phi_{n+1} & =\left(\psi_{1} \phi_{n}\right)^{+}+\left(\psi_{2} \chi_{n}\right)^{+}, \\
\chi_{n+1} & =\left(\psi_{3} \phi_{n}\right)^{-}+\left(\psi_{4} \chi_{n}\right)^{-}, \\
\tilde{\phi}_{n+1} & =\left(\psi_{1} \tilde{\phi}_{n}\right)^{+}+\left(\psi_{2} \tilde{\chi}_{n}\right)^{+}, \\
\tilde{\chi}_{n+1} & =\left(\psi_{3} \tilde{\phi}_{n}\right)^{-}+\left(\psi_{4} \tilde{\chi}_{n}\right)^{-} .
\end{aligned}
$$

Let $M=\max \left(\left\|\psi_{i}\right\| ; i=1,2,3,4\right)$. Then, it is not hard to show by induction that $\max \left(\left\|\phi_{n+1}\right\|,\left\|\chi_{n+1}\right\|,\left\|\tilde{\phi}_{n+1}\right\|,\left\|\tilde{\chi}_{n+1}\right\|\right) \leqq 2^{n}(M N)^{n+1}$. Thus, for values of $|s|<1 / 2 M N$, the generating functions

$$
\begin{aligned}
\phi & =\sum_{n=0}^{\infty} \phi_{n} s^{n}, & \tilde{\phi} & =\sum_{n=0}^{\infty} \tilde{\phi}_{n} s^{n}, \\
\chi & =\sum_{n=0}^{\infty} \chi_{n} s^{n}, & \tilde{\chi} & =\sum_{n=0}^{\infty} \tilde{\chi}_{n} s^{n},
\end{aligned}
$$

will converge to elements of $A$. It is also true that the generating functions in (2.2) are the unique solutions in $A$ of the equations (for $|s|<1 / 2 M N$ )

$$
\begin{aligned}
& \phi=s\left(\psi_{1} \phi\right)^{+}+s\left(\psi_{2} \chi\right)^{+}, \\
& \chi=1+s\left(\psi_{3} \phi\right)^{-}+s\left(\psi_{4} \chi\right)^{-}, \\
& \tilde{\phi}=1+s\left(\psi_{1} \tilde{\phi}\right)^{+}+s\left(\psi_{2} \tilde{\chi}\right)^{+}, \\
& \tilde{\chi}=s\left(\psi_{3} \tilde{\phi}\right)^{-}+s\left(\psi_{4} \tilde{\chi}\right)^{-} .
\end{aligned}
$$

We now define two matrices $P$ and $Q$ which will be important in all considerations to follow. Let

$$
P=\left[\begin{array}{cc}
P_{11} & P_{12} \\
P_{21} & P_{22}
\end{array}\right]=\left[\begin{array}{cc}
1-s \psi_{4} & -s \psi_{3} \\
0 & 1
\end{array}\right]\left[\begin{array}{ll}
\chi & \tilde{\chi} \\
\phi & \tilde{\phi}
\end{array}\right]
$$

and 


$$
Q=\left[\begin{array}{ll}
Q_{11} & Q_{12} \\
Q_{21} & Q_{22}
\end{array}\right]=\left[\begin{array}{cc}
1 & 0 \\
-s \psi_{2} & 1-s \psi_{1}
\end{array}\right]\left[\begin{array}{ll}
\chi & \tilde{\chi} \\
\phi & \tilde{\phi}
\end{array}\right]
$$

Using the Equations (2.3) and the Definitions (2.4) and (2.5) it is trivial to verify that

$$
P=\left[\begin{array}{rr}
1-s\left(\psi_{3} \phi\right)^{+}-s\left(\psi_{4} \chi\right)^{+} & -s\left(\psi_{3} \tilde{\phi}\right)^{+}-s\left(\psi_{4} \tilde{\chi}\right)^{+} \\
s\left(\psi_{1} \phi\right)^{+}+s\left(\psi_{2} \chi\right)^{+} & 1+s\left(\psi_{1} \tilde{\phi}\right)^{+}+s\left(\psi_{2} \tilde{\chi}\right)^{+}
\end{array}\right]
$$

and

$$
Q=\left[\begin{array}{rr}
1+s\left(\psi_{3} \phi\right)^{-}+s\left(\psi_{4} \chi\right)^{-} & s\left(\psi_{3} \tilde{\phi}\right)^{-}+s\left(\psi_{4} \tilde{\chi}\right)^{-} \\
-s\left(\psi_{1} \phi\right)^{-}-s\left(\psi_{2} \chi\right)^{-} & 1-s\left(\psi_{1} \tilde{\phi}\right)^{-}-s\left(\psi_{2} \tilde{\chi}\right)^{-}
\end{array}\right] .
$$

We can summarize these results into the following theorem:

Theorem 1. For sufficiently small $|s|$, the matrices $P$ and $Q$ defined in (2.4) and (2.5) satisfy

$$
P Q^{-1}=\frac{1}{1-s \psi_{1}}\left[\begin{array}{cc}
\left(1-s \psi_{1}\right)\left(1-s \psi_{4}\right)-s^{2} \psi_{2} \psi_{3} & -s \psi_{3} \\
s \psi_{2} & 1
\end{array}\right]
$$

where $1 /\left(1-s \psi_{1}\right)$ is defined by its geometric power series in s. Moreover, $P_{i j}$ and $Q_{i j}$ are power series in $s$ with constant terms $\delta_{i j}$ such that $P_{i j}-\delta_{i j}$ is in $A^{+}$and $Q_{i j}-\delta_{i j}$ is in $A^{-}$.

The matrix on the right in (2.6) will be denoted by $E$.

We now place the following condition $(U)$ on the operator $+:$ if $p_{i j}$ and $q_{i j}$ are power series in $s$ with positive radius of convergence having constant terms $\delta_{i j}$, if $p_{i j}-\delta_{i j}$ has coefficients from $A^{+}$and $q_{i j}-\delta_{i j}$ has coefficients from $A^{-}$, and if for some nonempty range $0 \leqq|s|<\epsilon$,

$$
\left(p_{i j}\right)\left(q_{i j}\right)^{-1}=E \text {, }
$$

then $p_{i j}$ is identically $P_{i j}$ and $q_{i j}$ is identically $Q_{i j}$. In other words, $E$ has a unique factorization of the type (2.7).

The following lemma contains a useful sufficient condition for $(U)$ to obtain. For more general results of this type in a slightly different setting see Wiener [4].

Lemma 1. If + is an idempotent operator, then condition $(U)$ holds in $A$.

Proof. First, we remark that for any $\psi$ in $A,\left(\psi^{-}\right)^{+}=0$. This comes directly from the equality $\psi^{+}=\left(\psi^{+}+\psi^{-}\right)^{+}=\left(\psi^{+}\right)^{+}+\left(\psi^{-}\right)^{+}=\psi^{+}+\left(\psi^{-}\right)^{+}$. We now write (2.7) in the form

$$
\left(p_{i j}\right)=E\left(q_{i j}\right)=\left(E_{i j}\right)\left(q_{i j}\right) .
$$

In some neighborhood of $s=0, E_{i j}$ is a convergent power series in $s$ with constant term $\delta_{i j}$. Let 


$$
\begin{gathered}
p_{i j}=\sum_{n=0}^{\infty} p_{i j}^{(n)} s^{n}, \quad q_{i j}=\sum_{n=0}^{\infty} q_{i j}^{(n)} s^{n}, \\
E_{i j}=\sum_{n=0}^{\infty} E_{i j}^{(n)} s^{n} .
\end{gathered}
$$

Suppose that for all $n=1,2, \cdots, N-1, p_{i j}^{(n)}$ and $q_{i j}^{(n)}$ have been uniquely determined from (2.8). Consider the coefficient of $s^{N}$ on both sides of $(2.8)$, that is,

$$
p_{i j}^{(N)}=\sum_{k=1}^{2} \sum_{n=0}^{N} E_{i k}^{(n)} q_{k j}^{(N-n)} .
$$

Using that $q_{k j}^{(n)}$ is known for $n \leqq N-1$, we can write

$$
p_{i j}^{(N)}=K_{i j}^{(N)}+\sum_{k=1}^{2} E_{i k}^{(0)} q_{k j}^{(N)}=K_{i j}^{(N)}+q_{i j}^{(N)}
$$

where $K_{i j}^{(M)}$ is known. By the remark at the beginning of the proof

$$
p_{i j}^{(N)}=\left[p_{i j}^{(N)}\right]^{+}=\left[K_{i j}^{(N)}\right]^{+}+\left[q_{i j}^{(N)}\right]^{+}=\left[K_{i j}^{(N)}\right]^{+},
$$

and

$$
q_{i j}^{(N)}=\left[K_{i j}^{(N)}\right]^{+}-K_{i j}^{(N)}=-\left[K_{i j}^{(N)}\right]^{-} .
$$

The proof of Lemma 1 follows by induction.

It would be very nice indeed at this point to write out explicit closed formulas for the elements $P_{i j}$ and $Q_{i j}$ in terms of $\psi_{i}(i=1,2,3,4)$. Unfortunately, we have not been able to do this. In fact even for the special operator + defined in $\$ 3$ we have not succeeded in doing this. Surprisingly enough, however, the results above are sufficiently powerful to enable one to compute in a few examples.

Wendel [5] has also used the Banach-algebra approach to fluctuation problems.

3. Changes of sign. Let $\left\{X_{k}\right\}$ be a sequence of independent, identically distributed random variables and let $S_{0}=0, S_{1}=X_{1}, \cdots, S_{n}=X_{1}+\cdots$ $+X_{n}$. We say a change of sign occurs between $S_{k}$ and $S_{k+1}$ if either $S_{k}>0$ and $S_{k+1} \leqq 0$, or $S_{k} \leqq 0$ and $S_{k+1}>0$. We also define the variable

$$
\tilde{N}_{n} \text { : the number of changes of sign in } S_{0}, S_{1}, \cdots, S_{n} \text {. }
$$

We wish to obtain information about the distribution of $\tilde{N}_{n}$ by the methods of $\S 2$.

Let $A$ denote the commutative Banach-algebra of functions

$$
\phi=\int_{-\infty}^{\infty} e^{i \ell x} d G(x)
$$


where $G(x)$ is of bounded variation on $-\infty<x<\infty$. The norm is just the total variation of $G(x)$ on $-\infty<x<\infty$. The product of two functions $\phi_{1}$ and $\phi_{2}$ in $A$ is, of course, the Fourier-Stieltjes transform of the convolution of their corresponding functions $G_{1}(x)$ and $G_{2}(x)$. For any $\phi$ of the form (3.1), let

$$
\phi^{+}=\int_{0^{+}}^{\infty} e^{i t x} d G(x), \quad \phi^{-}=\int_{-\infty}^{0^{+}} e^{i t x} d G(x) .
$$

Note that + is bounded, linear and idempotent. Thus, both Theorem 1 and Lemma 1 apply in this case. The norm $N$ of the operator + and the operator - is just 1 .

Now let

$$
\psi=\int_{-\infty}^{\infty} e^{i t x} d F(x), \quad(\|\psi\|=1),
$$

be the characteristic function of the variable $X_{k}$. Take $\psi_{1}=\psi_{4}=\psi$ and $\psi_{2}=\psi_{3}=u \psi(|u|<1)$ in the definition of the sequences given in (2.1). The generating functions will once again be denoted by $\phi, \chi, \tilde{\phi}$, and $\tilde{\chi}$. The results of $\$ 2$ state that in some neighborhood of $s=0$ the matrices

$$
P=\left[\begin{array}{cc}
1-s \psi & -s u \psi \\
0 & 1
\end{array}\right]\left[\begin{array}{cc}
\chi & \tilde{\chi} \\
\phi & \tilde{\phi}
\end{array}\right]
$$

and

$$
Q=\left[\begin{array}{cc}
1 & 0 \\
-\operatorname{su\psi } \psi & 1-s \psi
\end{array}\right]\left[\begin{array}{ll}
\chi & \tilde{\chi} \\
\phi & \tilde{\phi}
\end{array}\right]
$$

satisfy uniquely

$$
P Q^{-1}=\frac{1}{1-s \psi}\left[\begin{array}{cc}
(1-s \psi)^{2}-s^{2} u^{2} \psi^{2} & -s u \psi \\
s u \psi & 1
\end{array}\right] .
$$

The following lemma will tie loose ends together:

Lemma 2. Let $\psi$ be the characteristic function of $X_{k}$. Then, $\phi, \chi, \tilde{\phi}$, and $\tilde{\chi}$ converge for all $|s|<1$ and $|u|<1$. Moreover,

$$
\begin{gathered}
\phi=\sum_{n=0}^{\infty} \sum_{k=0}^{n} u^{k} s^{n} \int_{0^{+}}^{\infty} e^{i t x} d_{x} P\left\{\tilde{N}_{n}=k, S_{n}<x\right\}, \\
\chi=\sum_{n=0}^{\infty} \sum_{k=0}^{n} u^{k} s^{n} \int_{-\infty}^{0^{+}} e^{i t x} d_{x} P\left\{\tilde{N}_{n}=k, S_{n}<x\right\} .
\end{gathered}
$$

Proof. It is evident that $\phi$ and $\chi$ converge for all $|s|<1$ and $|u|<1$ if 
they are given by (3.4). Assume that for all $n=1,2, \cdots, K-1$ it has been shown that

$$
\phi_{n}=\sum_{k=0}^{n} u^{k} \int_{0^{+}}^{\infty} e^{i t x} d x P\left\{\tilde{N}_{n}=k, S_{n}<x\right\}
$$

and

$$
\chi_{n}=\sum_{k=0}^{n} u^{k} \int_{-\infty}^{0^{+}} e^{i t x} d_{x} P\left\{\tilde{N}_{n}=k, S_{n}<x\right\}
$$

Then,

$$
\begin{aligned}
\phi_{K}= & \left(\psi \phi_{K-1}\right)^{+}+u\left(\psi \chi_{K-1}\right)^{+} \\
= & \sum_{k=0}^{K-1} u^{k} \int_{0^{+}}^{\infty} e^{i t x} d_{x} \int_{0^{+}}^{\infty} F(x-y) d_{y} P\left\{\tilde{N}_{K-1}=k, S_{K-1}<y\right\} \\
& +u \sum_{k=0}^{K-1} u^{k} \int_{0^{+}}^{\infty} e^{i t x} d_{x} \int_{-\infty}^{0^{+}} F(x-y) d_{y} P\left\{\tilde{N}_{K-1}=k, S_{K-1}<y\right\} \\
= & \sum_{k=0}^{K-1} u^{k} \int_{0^{+}}^{\infty} e^{i t x} d_{x} P\left\{\tilde{N}_{K}=k, S_{K-1}>0, S_{K}<x\right\} \\
& +\sum_{k=0}^{K-1} u^{k+1} \int_{0^{+}}^{\infty} e^{i t x} d_{x} P\left\{\tilde{N}_{K}=k+1, S_{K-1} \leqq 0, S_{K}<x\right\} \\
= & \sum_{k=0}^{K} u^{k} \int_{0^{+}}^{\infty} e^{i t x} d_{x} P\left\{\tilde{N}_{K}=k, S_{K}<x\right\} .
\end{aligned}
$$

The demonstration of (3.6) for $n=K$ is analogous to the induction step shown above. Thus, (3.5) and (3.6) are valid for all $n$. To finish the proof of Lemma 2 we merely state without proof that

$$
\tilde{\phi}=1+\sum_{n=1}^{\infty} \sum_{k=1}^{n} u^{k-1} s^{n} \int_{0^{+}}^{\infty} e^{i t x} d_{x} P\left\{\tilde{N}_{n}=k, S_{n}<x\right\}
$$

and

$$
\tilde{\chi}=\sum_{n=1}^{\infty} \sum_{k=0}^{n-1} u^{k+1} s^{n} \int_{-\infty}^{0^{+}} e^{i t x} d_{x} P\left\{\tilde{N}_{n}=k, S_{n}<x\right\} .
$$

The convergence of $\tilde{\phi}$ and $\tilde{\chi}$ for $|s|<1$ and $|u|<1$ is immediate from (3.7) and (3.8).

At this time we summarize our results into a theorem.

THEOREM 2. Let $\psi$ be the characteristic function of $X_{k}$. Then, there exists a unique pair of matrices $P$ and $\widetilde{Q}$ whose elements $P_{i j}$ and $\widetilde{Q}_{i j}$ are power series in $s$ and $u$ which converge for $|s|<1$ and $|u|<1$, which have constant terms $\delta_{i j}$, 
and which have coefficients from $A^{+}$and $A^{-}$, respectively, such that for $|s|<1$ and $|u|<1$

$$
P \widetilde{Q}=\frac{1}{1-s \psi}\left[\begin{array}{cc}
(1-s \psi)^{2}-s^{2} u^{2} \psi^{2} & -s u \psi \\
s u \psi & 1
\end{array}\right] .
$$

Moreover,

$$
\begin{aligned}
& P_{21}=\sum_{n=0}^{\infty} \sum_{k=0}^{n} u^{k} s^{n} \int_{0^{+}}^{\infty} e^{i t x} d_{x} P\left\{\tilde{N}_{n}=k, S_{n}<x\right\}, \\
& \widetilde{Q}_{22}=\sum_{n=0}^{\infty} \sum_{k=0}^{n} u^{k} s^{n} \int_{-\infty}^{0^{+}} e^{i t x} d_{x} P\left\{\tilde{N}_{n}=k, S_{n}<x\right\} .
\end{aligned}
$$

Proof. Relation (3.3) holds in the neighborhood of $s=0$. Taking determinants of both sides of (3.3), we find

$$
|P|\left|Q^{-1}\right|=\left|P Q^{-1}\right|=1 \text {. }
$$

Because of the special properties of the + operator defined in (3.2) for the functions of type (3.1), $A^{+}$and $A^{-}$are both closed under multiplication. This implies that $|P|$ and $\left|Q^{-1}\right|$ are power series in $s$ with coefficients from $A^{+}$ and $A^{-}$, respectively. It is well-known (see, for example, [6], or [7, Chapter IV, Theorem 6.2]) that (3.10) uniquely determines $|P|$ and $\left|Q^{-1}\right|$ in this case. Necessarily $|P|=\left|Q^{-1}\right|=1$. Thus,

$$
Q^{-1}=\left[\begin{array}{rr}
Q_{22} & -Q_{12} \\
-Q_{21} & Q_{11}
\end{array}\right] \text {. }
$$

It follows from (3.11) and Lemma 2 that the elements of $P$ and $Q^{-1}$ converge and have the appropriate form for all $|s|<1$ and $|u|<1$. Since the elements of the matrix on the right in (3.3) also converge for $|s|<1$ and $|u|<1,(3.3)$ must hold for all $|s|<1$ and $|u|<1$. The uniqueness of matrices $P$ and $\widetilde{Q}$ which satisfy (3.9) follows directly from the uniqueness of matrices $P$ and $Q$ which satisfy (3.3) in the neighborhood of $s=0$. Finally, we note that $P_{21}=\phi$ and $\widetilde{Q}_{22}=Q_{11}=\chi$. An application of Lemma 2 finishes the proof of Theorem 2 .

It is convenient in computation to use the fact that $A^{+}$and $A^{-}$are closed under multiplication. For example, if $p$ and $\tilde{q}$ are matrices of the appropriate type for which

$$
p \tilde{q}=\left[\begin{array}{cc}
(1-s \psi)^{2}-s^{2} u^{2} \psi^{2} & -s u \psi \\
s u \psi & 1
\end{array}\right]
$$

and if $r$ and $t$ are elements given by

$$
r=\exp \{[-\log (1-s \psi)]+\}
$$

and 


$$
t=\exp \left\{[-\log (1-s \psi)]^{-}\right\}
$$

then $P=r p$ and $\tilde{Q}=t \tilde{q}$.

EXAMPLE 1. Symmetric exponential. Consider the sequence $\left\{X_{k}\right\}$ of independent random variables each of which has density $f(x)=\exp \{-|x|\} / 2$ $(-\infty<x<\infty)$. The characteristic function of $X_{k}$ is $\psi=1 /\left(1+t^{2}\right)$. A special property of $\psi$ which makes computation possible in this example is that $4 \psi^{+} \psi^{-}=\psi$

We begin by postulating an equality of the form

$$
\begin{aligned}
{\left[\begin{array}{cc}
1+A \psi^{+}+B \psi^{+^{2}}-\left(D \psi^{+}+E \psi^{+^{2}}\right) \\
C \psi^{+} & 1+F \psi^{+}
\end{array}\right] } & \cdot\left[\begin{array}{cc}
1+A \psi^{-}+B \psi^{-2} & -C \psi^{-} \\
D \psi^{-}+E \psi^{-2} & 1+F \psi^{-}
\end{array}\right] \\
= & {\left[\begin{array}{cc}
(1-s \psi)^{2}-s^{2} u^{2} \psi^{2} & -s u \psi \\
\operatorname{su\psi } & 1
\end{array}\right] }
\end{aligned}
$$

Let

$$
K=2 \frac{1-(1-s)^{1 / 2}}{s} .
$$

Then, (3.12) is valid if the constants $A, B, \cdots, F$ are chosen to be

$$
\begin{aligned}
& A=-2 s K-\frac{4 u^{2} s^{2} K^{4}}{16-u^{2} s^{2} K^{4}}, \\
& B=s^{2} K^{2}-(1-s)^{1 / 2} \frac{16 u^{2} s^{2} K^{4}}{16-u^{2} s^{2} K^{4}}, \\
& C=D=\frac{16 u s K^{2}}{16-u^{2} s^{2} K^{4}}, \\
& E=-4 u s+(1-s)^{1 / 2} \frac{64 u s K^{2}}{16-u^{2} s^{2} K^{4}}, \\
& F=\frac{4 u^{2} s^{2} K^{4}}{16-u^{2} s^{2} K^{4}} .
\end{aligned}
$$

It is easy to verify that $(1-s \psi)=\left(1-s K \psi^{+}\right)\left(1-s K \psi^{-}\right)$. Putting these facts together we can write, for example,

$$
\begin{aligned}
& P_{21}=\frac{1}{1-s K \psi^{+}} \cdot \frac{16 u s K^{2}}{16-u^{2} s^{2} K^{4}} \psi^{+}, \\
& Q_{11}=\widetilde{Q}_{22}=\frac{1}{1-s K \psi^{-}}\left(1+\frac{4 u^{2} s^{2} K^{4}}{16-u^{2} s^{2} K^{4}} \psi^{-}\right) .
\end{aligned}
$$

Setting $t=0$ and adding the two expressions in (3.13) gives the generating function for the number of changes of sign. We find 


$$
\sum_{n=0}^{\infty} \sum_{k=0}^{n} u^{k} s^{n} P\left\{\tilde{N}_{n}=k\right\}=\frac{1}{(1-s)^{1 / 2}} \frac{4+u s K^{2}}{4-u s K^{2}} .
$$

A simple inversion of (3.14) with respect to $u$ gives

$$
\sum_{n=0}^{\infty} s^{n} P\left\{\tilde{N}_{n}=k\right\}= \begin{cases}\frac{1}{(1-s)^{1 / 2}} & k=0, \\ \frac{2}{(1-s)^{1 / 2}}\left(\frac{s K^{2}}{4}\right)^{k} & k \neq 0 .\end{cases}
$$

Finally, inverting with respect to $s$, we get

$$
P\left\{\tilde{N}_{n}=k\right\}=\left\{\begin{array}{cl}
\left(\begin{array}{c}
2 n \\
n
\end{array}\right) \frac{1}{2^{2 n}} & k=0, \\
2\left(\begin{array}{c}
2 n \\
n-k
\end{array}\right) \frac{1}{2^{2 n}} & 1 \leqq k \leqq n .
\end{array}\right.
$$

Andersen [2] showed that for symmetric variables with continuous distributions the number of positive partial sums is independent of the distribution. If we let $\left\{X_{k}\right\}$ be a sequence of independent variables having the continuous and symmetric distribution given by $P\left\{X_{k}<x\right\}=(x+1) / 2$, $-1<x<1$, and if we let $\psi$ be the characteristic function of $X_{k}$, then

$$
\left.\left(\psi^{2} \psi^{+}\right)+\right]_{t=0}=\int_{0}^{1} \frac{d x}{2} \int_{-x}^{2}\left(\frac{1}{2}-\frac{|y|}{4}\right) d y=\frac{17}{48} .
$$

Thus, the coefficient of $s^{3} u^{3}$ in $P_{21}$, when $t=0$, would be in this case

$$
\left.\left.\left.\left(\psi\left(\psi \psi^{+}\right)^{-}\right)^{+}\right]_{t=0}=\left(\psi^{2} \psi^{+}\right)^{+}\right]_{t=0}-\left(\psi\left(\psi \psi^{+}\right)^{+}\right)^{+}\right]_{t=0}=17 / 48-5 / 16=1 / 24 .
$$

This last number differs from the value of $P\left\{\tilde{N}_{3}=3\right\}$ given above for Example 1, showing that the type of invariance demonstrated by Andersen does not hold for change of sign.

EXAmple 2. Bernoulli variables. Let $\left\{X_{k}\right\}$ be a sequence of independent Bernoulli variables with distributions $P\left\{X_{k}=1\right\}=p$ and $P\left\{X_{k}=-1\right\}=q$. The characteristic function in this example is

$$
\psi=p e^{i t}+q e^{-i t} .
$$

Because of the very special form of $\psi$, a computation can be made in this case. We first postulate the existence of a matrix factorization of the form

$$
\begin{aligned}
& {\left[\begin{array}{cc}
1+A e^{i t}+B e^{2 i t} & -\left(D e^{i t}+E e^{2 i t}\right) \\
C e^{i t} & 1+F e^{i t}
\end{array}\right]} \\
& \quad \cdot\left[\begin{array}{cc}
G+H e^{-i t}+I e^{-2 i t} & -\left(L+M e^{-i t}\right) \\
J+K e^{-i t} & N
\end{array}\right]=\left[\begin{array}{cc}
(1-s \psi)^{2}-s^{2} u^{2} \psi^{2} & -s u \psi \\
\operatorname{su\psi } & 1
\end{array}\right],
\end{aligned}
$$


where $A, B, \cdots, N$ are constants. It can be shown that a factorization of this type exists and that in particular

$$
C=\frac{u s p}{R^{2}-s^{2} u^{2} p q}, \quad N=\frac{R^{2}}{R^{2}-s^{2} u^{2} p q},
$$

where

$$
R=\frac{1+\left(1-4 s^{2} p q\right)^{1 / 2}}{2}
$$

In addition it may be easily verified that

$$
1-s \psi=\left(1-\frac{s p}{R} e^{i t}\right)\left(R-s q e^{-i t}\right) .
$$

Putting together (3.15) and (3.17), we find that

$$
P_{21}=\frac{1}{1-\frac{s p}{R} e^{i t}} \cdot \frac{u s p}{R^{2}-s^{2} u^{2} p q} e^{i t}
$$

and

$$
Q_{11}=\widetilde{Q}_{22}=\frac{1}{R-s q e^{-i t}} \cdot \frac{R^{2}}{R^{2}-s^{2} u^{2} p q} .
$$

Setting $t=0$ in the above and inverting with respect to $u$, we find

$$
\begin{gathered}
\sum_{n=0}^{\infty} s^{n} P\left\{\tilde{N}_{n}=2 k\right\}=\frac{1}{R-s q}\left(\frac{s^{2} p q}{R^{2}}\right)^{k}, \\
\sum_{n=0}^{\infty} s^{n} P\left\{\tilde{N}_{n}=2 k+1\right\}=\frac{1}{R-s p} \frac{s p}{R}\left(\frac{s^{2} p q}{R^{2}}\right)^{k} .
\end{gathered}
$$

The inversion of (3.18) is particularly elegant in the symmetric case. For, if $p=q=1 / 2$, by (3.16) and (3.18)

$$
\sum_{n=0}^{\infty} s^{n} P\left\{\tilde{N}_{n}=k\right\}=\frac{1}{\left(1-s^{2}\right)^{1 / 2}}\left[\left(\frac{1-\left(1-s^{2}\right)^{1 / 2}}{s}\right)^{k+1}+\left(\frac{1-\left(1-s^{2}\right)^{1 / 2}}{s}\right)^{k}\right] .
$$

Thus,

$$
P\left\{\tilde{N}_{n}=k\right\}=\frac{1}{2^{n}}\left(\begin{array}{c}
n \\
{[n-k / 2]}
\end{array}\right) \quad(k=0,1,2, \cdots, n) .
$$




\section{REFERENCES}

1. P. Erdös and G. A. Hunt, Changes of sign of sums of random variables, Pacific J. Math. vol. 3 (1953) pp. 673-687.

2. E. S. Andersen, On the fluctuations of sums of random variables, Math. Scand. vol. 1 (1953) pp. 263-285.

3. Frank Spitzer, $A$ combinatorial lemma and its application to probability theory, Trans. Amer. Math. Soc. vol. 82 (1956) pp. 323-339.

4. Norbert Wiener, On the factorization of matrices, Comment. Math. Helv. vol. 29 (1955) pp. 97-111.

5. J. G. Wendel, Spitzer's formula: a short proof, Proc. Amer. Math. Soc. vol. 9 (1958) pp. 905-908.

6. Glen Baxter, An operator identity, Pacific J. Math. vol. 8 (1958) pp. 649-663.

7. J. L. Doob, Stochastic processes, New York, John Wiley and Sons, Inc., 1953.

UNIVERSITY OF MINNESOTA,

Minneapolis, MinNesota 